\title{
Das Ansehen nicht nur der FMH steht auf dem Spiel
}

\author{
M. Geiser
}

a und in Übereinstimmung mit Art. 8 der Standesordnung der FMH.

b The history of medicine has never been a particularly attractive subject in medical education, and one reason for this is that it is so unrelievedly deplorable a story. For century after century, all the way into the remote millennia of its origins, medicine got along by sheer guesswork and the crudest sort of empiricism. It is hard to conceive of a less scientific enterprise among human endeavors. Virtually anything that could be thought up for the treatment of disease was tried out at one time or another, and, once tried, lasted decades or even centuries before being given up. It was in retrospect, the most frivolous and irresponsible kind of human experimentation, based on nothing but trial and error, and usually resulting in precisely that sequence. Bleeding, purging, cupping, administration of infusions of every known plant, solutions of every known metal, every conceivable diet including total fasting, most of these based on the weirdest imaginings about the cause of disease, concocted out of nothing but thin air - this was the heritage of medicine up until a little over a century ago. It is astounding that the profession survived so long, and got away with so much with so little outcry. Almost everyone seems to have been taken in. Evidently one had to be a born sceptic, like Montaigne, to see through the old nonsense; but even Montaigne, who wrote scathingly about illnesses caused by doctoring centuries before Ivan Illich, had little effect. Most people were convinced of the magical powers of medicine und put up with it. [8]

Korrespondenz:

Prof. Dr. med. Max Geiser

Gossetstrasse 49

CH-3084 Wabern
Wenn Standesorganisationen, Mitglieder von medizinischen Fakultäten, von Fachgesellschaften und der Schweizerischen Akademie der Medizinischen Wissenschaften Toleranz gegen die Rückkehr und den Vormarsch der Magie in der Medizin zeigen, bekommen sie Beifall von den Medien und Politikern, für die im Bereich von Krankheit und Gesundheit das Interesse an der Wahrheit nicht oben auf der Agenda steht. «Lasst den Gesundheitsmarkt durch bizarre Heilpraktiken blühen. Wir kümmern uns um die seriöse Medizin und die biologische Forschung und wollen unsere Energie nicht durch Auseinandersetzungen mit magischen und geistheilerischen ‘follies` verpuffen», mag die Ansicht von vielbeschäftigten klinisch, ambulant oder in der Forschung tätigen Ärzten lauten.

Im Gegensatz zur Mehrheit von Presse, Radio und Fernsehen scheuen sich die jeweiligen Redaktoren der Schweizerischen Ärztezeitung nicht, hin und wieder eine Satire über den Gesundheitsbasar und Kritik an den Repräsentanten der FMH, des Nationalfonds, der SAMW, der Sanitätsdirektorenkonferenz und des BAG und BSV erscheinen zu lassen.

Nach einer langen Phase von toleranter Anpassung an den «Wandel der Gesellschaft», der von den einen als Dekadenz und von den andern als erfreuliche Liberalisierung interpretiert wird, scheint ein frischer Wind aus der Romandie den bequemen Trend zur Resignation wegzublasen. Es ist erfreulich festzustellen, dass ein Praktiker [1] mehr als 50 Kollegen zusammenbrachte, die der Ansicht sind, dass magischer Leerlauf von der obligatorischen Grundversicherung ferngehalten werden sollte, gemäss der Forderung von Art. $32 \mathrm{KVG}^{\mathrm{a}}$, nach dem eine Leistung nur dann von der Grundversicherung übernommen werden darf, wenn sie wirksam, zweckmässig und wirtschaftlich ist und die Wirksamkeit wissenschaftlich bewiesen ist. Das Eintreten für die freie Arztwahl ist ebenfalls zu begrüssen. Die Unterteilung der Medizin in eine akademische, technische, alternative und Beziehungsmedizin wirkt nicht klärend, sondern eher verwirrend.

Ein zur Skepsis neigender Arzt wird mit einiger Berechtigung bedauern, dass Befürworter [2], und die Gegner [1] der Integration von magischen Praktiken in die solidarisch finanzierte Grundversicherung keine einzige der wichtigsten Publikationen erwähnt, die die Torheiten und Irrwege der Medizin behandeln und dass in der Kontroverse die Mängel des «Programms Evaluation Komplementärmedizin» PEK nicht dargestellt werden. Bei den lesenswerten Publikationen handelt es sich um «Das autistischundisciplinierte Denken in der Medizin und seine Ueberwindung» [3], um «Effectiveness and Efficiency in Health Services» [4], um die «Follies and Fallacies in Medicine» [5], um «Homöopathie kritisch betrachtet» [6], um «Les charlatans de la sante» [7] und vor allem um die «Medical Lessons from History» [8]. Denn diese kurze, eindrückliche Darstellung der Entstehung der modernen Medizin mit revolutionärer Wirkung, sollte zur Pflichtlektüre jedes Medizinstudenten, Arztes, Gesundheitspolitikers und Journalisten erhoben werden, damit die Öffentlichkeit endlich die jämmerliche Wirkungslosigkeit der Heilkunde bis zu Beginn des 19. Jahrhunderts und die Tatsache zur Kenntnis nimmt, dass die Revolutionierung der Medizin zur effektvollen biomedizinischen Wissenschaft durch die Beseitigung von Dogmata vollzogen wurde. Ein kleiner Ausschnitt aus dieser klärenden Analyse soll dem Leser meines Plädoyers für eine vernünftige Medizin nicht vorenthalten werden. ${ }^{\mathrm{b}}$

Die Verwendung von Umfragenergebnissen als Argument für eine Integrierung von sogenannt komplementärmedizinischen Praktiken [2] ist tendenziös, weil Umfragen bekanntlich nicht die Wahrheit erforschen, sondern lediglich die momentane Meinung eines Teils einer Bevölkerung kundtut, aber für den geforderten wissenschaftlichen Beweis der Wirksamkeit nicht taugen. Darüber hinaus muss zur Kenntnis genommen werden, dass das Wünschbare keine Grenzen hat, wenn es um Gesundheit, Leben und Sicherheit geht und dass die Meinung einer Bevölkerung durch die von Medien praktizierte Desinformation und Angstmacherei im Bereich von Gesundheit und Krankheit beeinflusst wird. 


\section{Das Programm Evaluation Komplementärmedizin PEK - eine peinliche Alibiübung}

Die Öffentlichkeit weiss offenbar wenig oder nichts über das PEK. Im folgenden versuche ich, diese Informationslücke in kürzest möglicher Form zu schliessen.

Das von 1992 bis 1998 unter Einsatz von 6 Millionen Franken durchgeführte Nationale Forschungsprogramm «NFP 34 Komplementärmedizin» konnte keinen Wirksamkeitsnachweis für die geprüften komplementärmedizinisch genannten Heilpraktiken zeigen. Die vom Gesundheitsökonomen [9] durchgeführte Untersuchung zeigte, dass diese Praktiken meist zusätzlich und nicht an Stelle der konventionellen Medizin benutzt wurden und somit keine Kosten sparen, sondern sie erhöhen. Das negative Ergebnis des NFP 34 konnte nicht verhindern, dass fünf paramedizinische Heilpraktiken (Anthroposophische Medizin, Homöopathie, Neuraltherapie, Phytotherapie und die Traditionelle Chinesische Medizin TCM) provisorisch in die Grundversicherung aufgenommen wurden. Der Bundesrat gab unter Einsatz von erneut 6 Millionen Franken eine Untersuchung dieser fünf Praktiken auf ihre Wirksamkeit in Auftrag. Das 1999 begonnene und 2005 enden sollende, vom Bundesamt für Sozialversicherung BSV durchgeführte PEK kann trotz des auffällig grossen Einsatzes von 30 Akademikern aus 8 in- und ausländischen Universitäten (mit einem Lenkungsausschuss, Experten und Review Board) mit Hilfe von Fragebogen, Telefoninterviews und Konsultationsprotokollen in 2600 Arztpraxen in Kenntnis der Mängel an Verlässlichkeit, Kontrollierund Reproduzierbarkeit der verwendeten Untersuchungsmethode mit Umfragen lediglich etwas über die Beanspruchung der fünf Heilsysteme durch einen Teil der Schweizer Bevölkerung aussagen. Aber die Wirksamkeit dieser Praktiken lässt sich damit nicht prüfen. Die gleichzeitig mit der Umfrage durchgeführte Literaturstudie ist überflüssig, da zahlreiche randomisierte, kontrollierte, die Wirksamkeit prüfende Studien und Metaanalysen [10] lediglich eine vorübergehende, suggestive Beeinflussung der Befindlichkeit im Sinne einer Placebowirkung zeigen konnten. Spezifische Wirkungen fehlen, auf die nicht verzichtet werden könnte oder den natürlichen «Grossmuttermethoden» in Form von verständnisvoller, beruhigender Zuwendung, Trost, Tees, Salben und Wickeln überlegen wären. Im Gegensatz zu diesen für jedermann erschwinglichen und den indizierten Einsatz wirkungsvoller Medizin nicht behindernden Hausmittelchen verwendet die sich irreführenderweise Naturheilkunde nennenden Phantastereien eine unbegrenzte Zahl von Placebos, die nicht ohne Nebenwirkungen sind, Heilung bei heilbaren Krankheiten verhindern oder verzögern und bei noch unheilbaren Leiden falsche Hoffnungen wecken können und das irrationale Denken unterstützen.

Das PEK tangiert wegen seiner fehlenden Notwendigkeit und insbesondere wegen der verwendeten pseudowissenschaftlichen Methoden zur Wirksamkeitsprüfung das Ansehen des durchführenden Instituts für «Evaluative Forschung in der Orthopädie», der zugehörigen Universität Bern und des für das PEK verantwortlichen Bundesamtes für Sozialversicherung BSV und des Eidgenössischen Departements des Innern EDI.

\section{Freier Markt für alternativ und komplementär genannte heilerische Praktiken}

Es ist selbstverständlich Bürgerinnen und Bürgern, die sich auch im Gesundheitsbereich selbstverwirklichen können sollen, unbenommen, Praktiken, die auf dem irreführenden Glauben an magische Kräfte beruhen und Heilung verheissen, auf eigene Verantwortung anzuwenden und selbst zu finanzieren. Wer sich auf dem Gesundheitsmarkt irreführen lassen will, braucht keine solidarische Unterstützung. Denn wer sich diese Praktiken nicht leisten kann, darf sich nicht über fehlende Solidarität beklagen. Er muss sich im Gegenteil dafür bedanken, vor Leerlauf, Irreführung und Ausbeutung bewahrt worden zu sein. Die kürzlich gestartete Initiative «Ja zur Komplementärmedizin» entspricht einem auf Unwissen und Desinformation beruhenden Schildbürgerstreich in Form der Rückstufung der Heilkunde auf das Niveau von vorwissenschaftlichen Phantastereien, die bis ins 19. Jahrhundert auch die diplomierten Ärzte beherrschte.

\section{Schlussfolgerungen}

Es geht bei der Kontroverse für oder wider die Integration der sogenannten Komplementärmedizin in die solidarisch finanzierte, obligatorische Grundversicherung um die Beurteilung des Einflusses auf die Wirksamkeit und Kosten des Gesundheitswesens. Es gibt plausible Hinweise dafür, dass die Integration die Wirksamkeit senkt $[10,11]$ und die Kosten erhöht [9]. Somit wäre es vernünftig, die KM dem freien Markt zu überlassen und damit die kostenbegrenzende Selbst- 
finanzierung einzuführen. Es gibt noch andere Leerlaufleistungen im geltenden Leistungskatalog, die mit fragwürdiger Begründung ganz oder teilweise solidarisch finanziert werden, z.B. im Vorsorge-, Nachbehandlungs- und Chiropraxisbereich. Mit der Integration von medizinischen Leistungen ohne Wirksamkeitsnachweis in die solidarisch finanzierte Grundversicherung steht die Glaubwürdigkeit der Heilkunde und des ärztlichen Berufsstandes auf dem Spiel. Die mit untauglichen Methoden durchgeführte Wirksamkeitsprüfung durch das PEK trägt nichts zum Ansehen bei. Deswegen drängt sich die Distanzierung von magischen Phantastereien in der Heilkunde und einer überflüssigen Wirksamkeitsprüfung mit untauglichen Methoden aus den gleichen Gründen auf wie die in der «Erklärung zur Homöopathie» vor 12 Jahren von 36 Fachleuten unterzeichnete Distanzierung von der Homöopathie als einer Irrlehre [15], die sich vergeblich [12-14] wissenschaftliche Anerkennung verschaffen wollte. Es geht erneut darum, die Flut von Fehlinformationen über widernatürliche, magischem Denken entspringende Praktiken aufzuhalten und den späteren Vorwurf zu vermeiden, dass sich niemand zu Wort gemeldet und den Mut gehabt habe, magische Phantastereien beim Namen zu nennen und ihre Integration ins solidarisch finanzierte Gesundheitswesen als gesundheitspolitischen Schildbürgerstreich mit Irreführung des Publikums zu bezeichnen.

\section{Literatur}

1 Valloton P. Soll die FMH unter ihren Mitgliedern das komplementärmedizinische Angebot fördern? Schweiz Ärztezeitung 2005,86:24-7.

2 Fritschi J. Soll sich die FMH für die Erhaltung oder sogar für die Förderung komplementärmedizinischer Angebote bei ihren Mitgliedern einsetzen? Schweiz Ärztezeitung 2005,86:27-30.

3 Bleuler E. Das autistisch-undisciplinierte Denken in der Medizin und seine Ueberwindung. Berlin: Springer; 1927.

4 Cochrane AL. Effectiveness and Efficiency. Random Reflexions on Health Services Provincial Hospitals Trust. Abingdon: Burgers and Son; 1972.

5 Skrabanek P, McCormick J. Follies and Fallacies in Medicine. Glasgow: The Terragone Press; 1989.

6 Hopff WH. Homöopathie kritisch betrachtet. Stuttgart, New York: Thieme Verlag; 1991.

7 Abgrall JM. Les charlatans de la santé. Paris: Documents Payot; 1998.

8 Thomas L. Medical Lessons from History in «The medusa and the snail». New York: The Viking Press; 1979. S. 158-75.

9 Sommer JH, Bürgi M, Theiss R. A randomized experiment of the effects of including alternative medicine in the mandatory benefit package of health insurance funds in Switzerland. Compl Ther Med 1999;7:54-61.

10 Ernst E. The Desktop Guide to Complementary and Alternative Medicine. Edinburgh, London, New York, St. Louis, Sydney, Toronto: Mosby; 2001.

11 Ernst E. Patient choice and complementary medicine. J Roy Soc Med 2004;97:41.

12 Davenas E, et al.: Human basophil degranulation triggered by very dilute antiserum against JgE. Nature 1988;333:816-8.

13 Maddox J, Randi J, Steward WH. High dilution experiments a delusion. Nature 1988;334:287-90.

14 Hirst SJ, Hayes NA, Burridge J, Pearce FL, Foreman JC. Human basophil degranulation is not triggered by very dilute antiserum against human IGE. Nature 1993;366:525-7.

15 Prokop O, Hopff WH. Gibt es heute noch Schildbürgerstreiche? Schweiz Med Wochenschr 1992; 122:770-9. 